01

\title{
Изотопные эффекты в спектрах комплексов с водородной связью. Экспериментальное и теоретическое изучение спектра ИК поглощения комплекса $\left({ }^{12} \mathrm{CH}_{3}\right){ }_{2}^{13} \mathrm{CO}$. . . HF
}

\author{
(C) В.П. Булычев, Е.А. Енгалычева, К.Г. Тохадзе
}

Санкт-Петербургский государственный университет, 199034 Санкт-Петербург, Россия

e-mail: v.bulychev@spbu.ru; e-mail: k.tokhadze@spbu.ru

Поступила в редакцию 26.11.2018 г.

В окончательной редакции 26.11.2018 г.

Принята к публикации 04.12.2018 г.

\begin{abstract}
ИК спектры поглощения смесей $\left({ }^{12} \mathrm{CH}_{3}\right){ }_{2}^{13} \mathrm{CO} / \mathrm{HF}$ и свободных молекул $\left({ }^{12} \mathrm{CH}_{3}\right){ }_{2}^{13} \mathrm{CO}$ зарегистрированы в области 4000-800 $\mathrm{cm}^{-1}$ с использованием вакуумного фурье-спектрометра Bruker IFS-125 HR при комнатной температуре с разрешением $0.05 \mathrm{~cm}^{-1}$. Полосы поглощения комплекса $\left({ }^{12} \mathrm{CH}_{3}\right)_{2}^{13} \mathrm{CO} \ldots \mathrm{HF}$ получены вычитанием полос поглощения свободных молекул НF и ацетона и линий поглощения атмосферной воды из экспериментального спектра смесей. Спектральные характеристики обертона $2 v\left({ }^{13} \mathrm{C}=\mathrm{O}\right)$ свободного ацетона также зарегистрированы. Сравнение полученных спектральных данных с аналогичными данными, измеренными ранее для комплекса $\left({ }^{12} \mathrm{CH}_{3}\right){ }_{2}^{12} \mathrm{CO} \ldots \mathrm{HF}$, показывает изменения в спектрах поглощения комплексов, вызванные изотопным замещением ${ }^{12} \mathrm{C} \rightarrow{ }^{13} \mathrm{C}$. Частоты и интенсивности полос поглощения обоих комплексов рассчитываются по теории возмущений с использованием методов $\mathrm{MP} 2 / 6-311++\mathrm{G}(2 \mathrm{~d}, 2 \mathrm{p})$ и MP2/6-311++G(3df,3pd) с учетом ошибки наложения базисных функций мономеров. Результаты расчетов находятся в хорошем согласии с экспериментальными данными и используются при интерпретации наблюдаемых спектров. Влияние ангармонических эффектов на частоты и интенсивности наиболее интенсивных полос $v(\mathrm{H}-\mathrm{F})$ и $v\left({ }^{13} \mathrm{C}=\mathrm{O}\right)$ изучено при помощи вариационных расчетов. Показано, что в отличие от комплекса $\left({ }^{12} \mathrm{CH}_{3}\right){ }_{2}^{12} \mathrm{CO} \ldots \mathrm{HF}$ в комплексе $\left({ }^{12} \mathrm{CH}_{3}\right){ }_{2}^{13} \mathrm{CO} \ldots \mathrm{HF}$ резонанс $2 v\left({ }^{13} \mathrm{C}=\mathrm{O}\right) / v(\mathrm{H}-\mathrm{F})$ практически отсутствует.
\end{abstract}

DOI: $10.21883 /$ OS.2019.04.47507.341-18

\section{Введение}

Изотопное замещение в молекулярных комплексах с водородной связью может приводить к значительным изменениям колебательной динамики этих систем, значений момента инерции и вращательных постоянных, частот и интенсивностей переходов между колебательными состояниями и средних значений геометрических параметров [1]. Эти эффекты объясняются влиянием изотопного замещения на приведенные массы и формы колебаний, а также на их амплитуды. Так как величина внутримодовых и межмодовых ангармонических взаимодействий зависит от амплитуд и формы колебаний, то влияние ангармоничности на структурные и спектральные параметры комплексов может меняться при изотопном замещении. Изотопные эффекты в спектрах ИК поглощения молекулярных комплексов приводят к смещению колебательных полос и изменению их интегральной интенсивности и формы контура.

Экспериментальное и теоретическое изучение спектров поглощения комплексов с водородной связью различных изотопологов позволяет лучше понять колебательную динамику этих систем, величину ангармонического взаимодействия между отдельными колебаниями и механизм формирования экспериментально наблюда- емых полос поглощения [2]. В частности, в [2] было показано, что изотопное замещение значительно сильнее влияет на интенсивности спектральных переходов, чем на частоты. Одновременное экспериментальное и теоретическое изучение комплексов, образованных молекулами галоидоводородов с различными молекулами акцепторов протона, может дать богатую информацию о природе явления межмолекулярной водородной связи.

Комплексы фтористого водорода с молекулами воды, диметилового эфира и ацетона являются удобными объектами для таких исследований, так как экспериментальные спектры имеют детальную структуру, а геометрические и спектроскопические параметры комплексов могут быть достаточно точно рассчитаны современными квантово-механическими методами [3].

Водородно-связанные комплексы галоидоводородов с ацетоном не были столь подробно изучены, как комплексы с молекулами воды. ИК спектры комплексов $\left({ }^{12} \mathrm{CH}_{3}\right){ }_{2}^{12} \mathrm{CO} \ldots$. HF в газовой фазе были зарегистрированы с низким разрешением в области $3900-3200 \mathrm{~cm}^{-1}$ в [4] и в области валентного и либрационного колебаний HF в [5]. Спектры поглощения комплексов $\left({ }^{12} \mathrm{CH}_{3}\right){ }_{2}^{12} \mathrm{CO} \ldots \mathrm{HF}(\mathrm{DF})$ и $\left({ }^{12} \mathrm{CD}_{3}\right){ }_{2}^{12} \mathrm{CO} \ldots \mathrm{HF}(\mathrm{DF})$ в области $3400-400 \mathrm{~cm}^{-1}$ были зарегистрированы в аргоновой матрице при $12 \mathrm{~K}$ [6]. В работе [3] спектры 
поглощения комплексов $\left({ }^{12} \mathrm{CH}_{3}\right){ }_{2}^{12} \mathrm{CO} \ldots \mathrm{HF}$ и их полностью или частично дейтерированных изотопологов были измерены при комнатной температуре в области $3900-3200 \mathrm{~cm}^{-1}$ с разрешением $0.02-0.2 \mathrm{~cm}^{-1}$. В [7] спектры поглощения газовой смеси $\left({ }^{12} \mathrm{CH}_{3}\right){ }_{2}^{12} \mathrm{CO} / \mathrm{HF}$ и свободных молекул ацетона $\left({ }^{12} \mathrm{CH}_{3}\right){ }_{2}^{12} \mathrm{CO}$ были зарегистрированы в более широком диапазоне 4000-900 $\mathrm{cm}^{-1}$ при комнатной температуре с разрешением $0.02 \mathrm{~cm}^{-1}$ при давлениях 7-22 (HF) и 9-120 Torr (ацетон). Была надежно зарегистрирована полоса обертона $2 v(\mathrm{C}=\mathrm{O})$ свободного ацетона в области $3450 \mathrm{~cm}^{-1}$, т. е. в области полосы $v(\mathrm{H}-\mathrm{F})$ комплекса. Контуры полос $v(\mathrm{H}-\mathrm{F})$ и $v(\mathrm{C}=\mathrm{O})$ комплекса $\left({ }^{12} \mathrm{CH}_{3}\right)_{2}^{12} \mathrm{CO} \ldots \mathrm{HF}$ были получены вычитанием полос поглощения свободных молекул НF и ацетона и линий поглощения атмосферной воды из экспериментальных спектров газовых смесей.

Равновесная геометрия комплекса $\left(\mathrm{CH}_{3}\right)_{2} \mathrm{CO} \ldots \mathrm{HF}$ была рассчитана в $[8,9]$ в приближении Хартри-Фока, и структура спектра поглощения в области полосы $v(\mathrm{H}-\mathrm{F})$ была интерпретирована на основе решения двумерной задачи взаимодействующих валентных колебаний связи $\mathrm{H}-\mathrm{F}$ и водородного мостика. В работе [3] равновесная ядерная конфигурация этого комплекса, а также поверхности потенциальной энергии и дипольного момента были рассчитаны на более высоком $a b$ initio уровне с учетом корреляции в движении электронов и ошибки наложения наборов базисных функций мономеров. Частоты и абсолютные интенсивности фундаментального $v(\mathrm{H}-\mathrm{F})$ перехода и суммарных и разностных переходов, включающих возбужденные состояния валентного колебания Н-связи, были определены из вариационного решения двумерного колебательного уравнения. Сравнение этих данных с аналогичными результатами расчетов родственных комплексов HF с молекулами воды и диметилового эфира позволило объяснить, почему плечи экспериментально наблюдаемой полосы $v(\mathrm{H}-\mathrm{F})$ имеют максимальную интенсивность в спектре комплекса НF. . ацетон. Колебания комплекса $\left({ }^{12} \mathrm{CH}_{3}\right){ }_{2}^{12} \mathrm{CO}$... HF были более тщательно рассчитаны в [7]. Помимо одномерных ангармонических задач были решены двумерные, трехмерные и четырехмерные задачи ангармонического взаимодействия валентных колебаний $\mathrm{C}=\mathrm{O}, \mathrm{H}-\mathrm{F}$ и Н-связи с либрационными колебаниями мономеров в плоскости комплекса. Ангармонические задачи решались вариационным методом с использованием потенциальной энергии и дипольного момента, рассчитанных в приближении МР2/6-311++G(2d,2p) с учетом ошибки наложения базисных наборов мономеров. Эти расчеты предсказали, в частности, наличие в комплексе сильного резонанса между основным тоном колебания $v(\mathrm{H}-\mathrm{F})$ и обертоном колебания $\mathrm{C}=\mathrm{O}$, что приводит к значительному увеличению интенсивности обертона.

Целью настоящей работы является регистрация ИК спектров поглощения комплексов $\left({ }^{12} \mathrm{CH}_{3}\right){ }_{2}^{13} \mathrm{CO} \ldots \mathrm{HF}$ и свободных молекул $\left({ }^{12} \mathrm{CH}_{3}\right)_{2}^{13} \mathrm{CO}$ в газовой фазе при комнатной температуре с разрешением $0.05 \mathrm{~cm}^{-1}$ и сравнение этих спектров со спектрами $\left({ }^{12} \mathrm{CH}_{3}\right){ }_{2}^{12} \mathrm{CO} \ldots$. HF и $\left({ }^{12} \mathrm{CH}_{3}\right){ }_{2}^{12} \mathrm{CO}$, полученными в [7]. Для интерпретации экспериментальных данных частоты и интенсивности спектральных переходов между колебательными состояниями комплексов и свободных молекул ацетона были рассчитаны в гармоническом и ангармоническом приближениях при помощи пакета программ GAUSSIAN 16 [10]. Ангармонические расчеты спектральных параметров комплексов были выполнены в пространстве нормальных колебаний с учетом взаимодействия всех колебательных мод, используя метод колебательной теории возмущений второго порядка [1113]. Были проанализированы изменения в спектрах ацетона и фтористого водорода, вызванные образованием комплекса, и изменения в спектрах молекул и комплекса, вызванные изотопным замещением. Вариационный метод был использован для изучения межмодового ангармонического взаимодействия, в частности для изучения возможности резонанса между обертоном колебания ${ }^{13} \mathrm{C}=\mathrm{O}$ и основным тоном валентного колебания $\mathrm{H}-\mathrm{F}$.

\section{Методика эксперимента}

ИК спектры свободных молекул изотопозамещенного ацетона $\left({ }^{12} \mathrm{CH}_{3}\right){ }_{2}^{13} \mathrm{CO}$, фтористого водорода $\mathrm{HF}$ и их газовых смесей $\left({ }^{12} \mathrm{CH}_{3}\right){ }_{2}^{13} \mathrm{CO} / \mathrm{HF}$ были получены при температуре $295 \mathrm{~K}$. Особое внимание было уделено регистрации и анализу спектров в области полос поглощения валентных колебаний $v(\mathrm{H}-\mathrm{F})$ и $v(\mathrm{C}=\mathrm{O})$ партнеров. Для сравнения были также зарегистрированы спектры смесей $\left({ }^{12} \mathrm{CH}_{3}\right){ }_{2}^{12} \mathrm{CO} / \mathrm{HF}$. Использовались кюветы из нержавеющей стали длиной $10 \mathrm{~cm}$ с окнами из лейкосапфира (область пропускания до $1800 \mathrm{~cm}^{-1}$ ) и $\mathrm{ZnSe}$ (область пропускания до $800 \mathrm{~cm}^{-1}$ ), поверхности которых имеют оптический клин, что позволяет подавить интерференцию. Необходимо отметить, что наиболее подходящими для работы с HF являются окна из сапфира, однако необходимость регистрировать спектры в области полос поглощения $v(\mathrm{C}=\mathrm{O})$ и ниже заставляет использовать менее инертные окна из $\mathrm{ZnSe}$.

Откачка кювет и измерение давления образцов проводились с помощью специальной системы из нержавеющей стали. Для измерения давления использовался мановакуумметр „Blondelle SA“ из нержавеющей стали. Поскольку количество изотопозамещенного ацетона $\left({ }^{12} \mathrm{CH}_{3}\right){ }_{2}^{13} \mathrm{CO}$ было невелико, был реализован минимальный объем измерительной части системы. Спектры регистрировались с разрешением от 0.1 до $0.05 \mathrm{~cm}^{-1}$ при суммарных давлениях смеси $\left(\mathrm{CH}_{3}\right)_{2} \mathrm{CO} / \mathrm{HF} 30-100$ Torr. Полосы поглощения комплексов получены вычитанием поглощения свободных молекул НF, ацетона и атмосферной воды из экспериментальных спектров смесей. Хотя в исследуемом комплексе тонкая структура полос поглощения не наблюдается, тем не менее регистрация спектров в системе ацетон/HF с высоким разрешени- 


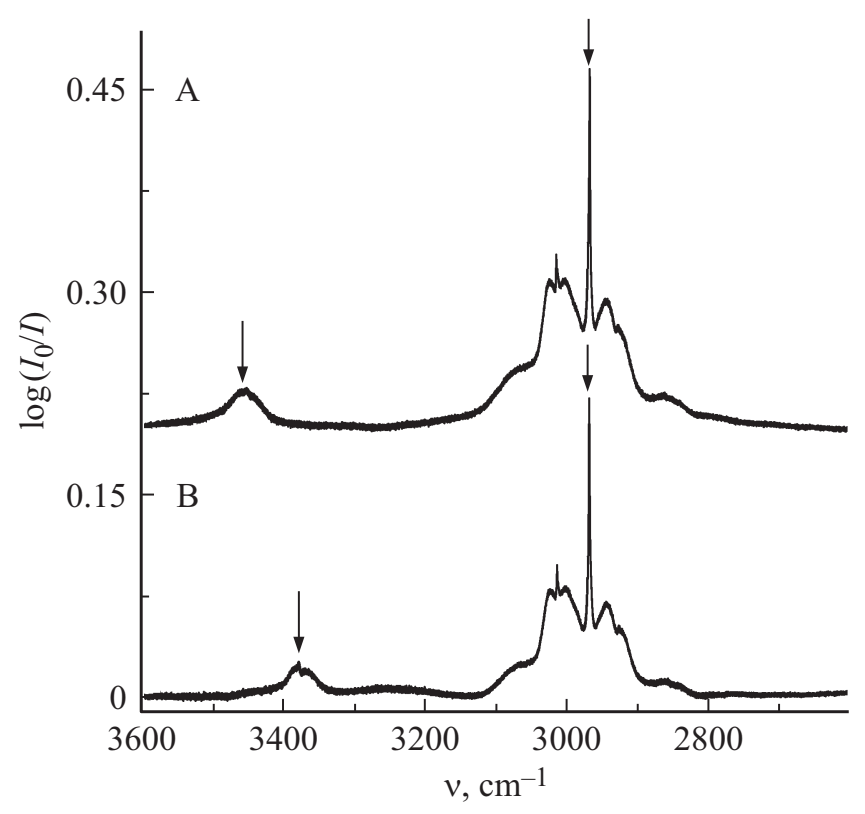

Рис. 1. Экспериментальные спектры поглощения свободных молекул ацетона в области валентных колебаний $\mathrm{CH}$ при давлении 20 Torr. $\mathrm{A}-\left({ }^{12} \mathrm{CH}_{3}\right){ }_{2}^{12} \mathrm{CO}, \mathrm{B}-\left({ }^{12} \mathrm{CH}_{3}\right){ }_{2}^{13} \mathrm{CO}$. Стрелками отмечены частоты полос, представленных в табл. 1.

ем до $0.05 \mathrm{~cm}^{-1}$ заметно упрощает задачу вычитания поглощения линий $\mathrm{HF}$ и $\mathrm{H}_{2} \mathrm{O}$ из измеряемого спектра. Важно, что выбранные условия эксперимента и процедуры обработки экспериментальных данных позволили нам получить спектры комплексов не только в области очень интенсивной полосы $\mathrm{H}-\mathrm{F}$, но и в окрестности менее интенсивных полос валентных колебаний $\mathrm{C}=\mathrm{O}$ и $\mathrm{C}-\mathrm{C}$ и деформационных колебаний $\mathrm{H}-\mathrm{C}-\mathrm{H}$.

Газовая кювета с необходимой смесью помещалась в один из двух каналов спектрометра, затем объем кюветного отделения откачивался. Предварительно регистрировался спектр пустой кюветы $\left(I_{0}\right)$ в откачанном кюветном отделении. В среднем регистрировалось 100 спектров пустой кюветы и 100 спектров кюветы с газовой смесью. В ряде измерений дополнительно регистрировался спектр кюветного отделения без кюветы. Очевидно, что общее число сканов во всех случаях можно варьировать как для улучшения отношения сигнал/шум, так и для уменьшения времени регистрации спектров.

Спектры свободных молекул и комплексов были зарегистрированы при давлении HF до 30 Torr и давлении ацетона 10-60 Torr. Экспериментальные спектры свободных молекул $\left({ }^{12} \mathrm{CH}_{3}\right){ }_{2}^{13} \mathrm{CO},\left({ }^{12} \mathrm{CH}_{3}\right){ }_{2}^{12} \mathrm{CO}$ и комплексов $\left({ }^{12} \mathrm{CH}_{3}\right){ }_{2}^{13} \mathrm{CO} . . \mathrm{HF},\left({ }^{12} \mathrm{CH}_{3}\right){ }_{2}^{12} \mathrm{CO}$. . . НF представлены на рис. $1-4$. Следует отметить, что в этой работе мы приводим более полные спектры свободного ацетона $\left({ }^{12} \mathrm{CH}_{3}\right){ }_{2}^{12} \mathrm{CO}$ и его комплекса с $\mathrm{HF}$, чем в [7], где спектры поглощения показаны только в областях полос валентных колебаний $\mathrm{H}-\mathrm{F}$ и $\mathrm{C}=\mathrm{O}$. В случае широких и сложных полос в качестве их характеристик целесооб- разно использовать величины спектральных моментов и прежде всего величину первого спектрального момента

$$
M_{1}=M_{0}^{-1} \int_{\text {over the band }} v D(v) d v
$$

т.е. значение центра тяжести полосы, где $M_{0}-$ интегральная интенсивность полосы и $D(v)$ - оптическая плотность. Частоты полос поглощения и значения их центров тяжести приведены в табл. 1.

Изотопозамещение ${ }^{12} \mathrm{C} \rightarrow{ }^{13} \mathrm{C}$ в группе $\mathrm{C}=\mathrm{O}$ молекулы ацетона приводит к заметным изменениям ряда полос в наблюдаемых спектрах поглощения свободных молекул ацетона и смесей ацетон/НF. В качестве иллюстрации наблюдаемых изменений на рис. 1 показана полоса поглощения обертона $2 v(\mathrm{C}=\mathrm{O})$ чистого ацетона. На рис. 2 видно, что в спектрах поглощения газовых смесей полоса обертона $2 v(\mathrm{C}=\mathrm{O})$ свободных молекул $\left({ }^{12} \mathrm{CH}_{3}\right){ }_{2}^{12} \mathrm{CO}$ попадает в область наиболее интенсивного максимума сложной полосы $v(\mathrm{H}-\mathrm{F})$ комплекса около $3450 \mathrm{~cm}^{-1}$, а полоса обертона свободных молекул $\left({ }^{12} \mathrm{CH}_{3}\right){ }_{2}^{13} \mathrm{CO}$ наблюдается в области низкочастотного крыла этой полосы около $3380 \mathrm{~cm}^{-1}$. Поглощение, связанное с валентными колебаниями группы $\mathrm{CH}_{3}$ (в области $\left.3000 \mathrm{~cm}^{-1}\right)$, практически не изменяется при изотопозамещении ${ }^{12} \mathrm{C} \rightarrow{ }^{13} \mathrm{CO}$ (рис. 1). Интенсивные полосы поглощения валентных колебаний $\mathrm{C}=\mathrm{O}\left(1750 \mathrm{~cm}^{-1}\right)$ и $\mathrm{C}-\mathrm{C}\left(1200 \mathrm{~cm}^{-1}\right)$ заметно сдвигаются в области более низких частот.

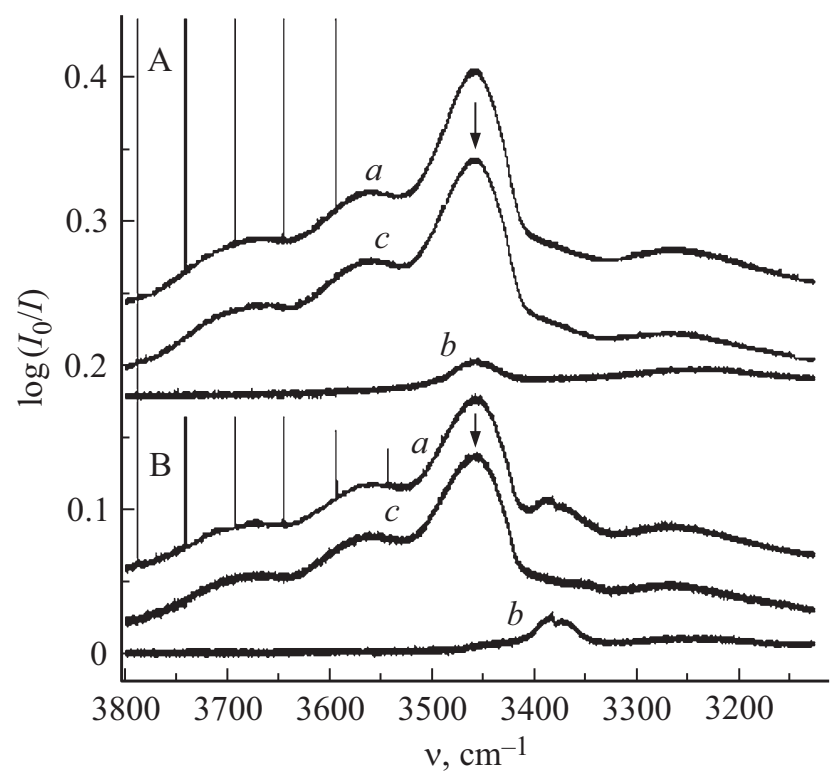

Рис. 2. Спектры поглощения свободных молекул $\left(\mathrm{CH}_{3}\right)_{2} \mathrm{CO}$ и комплексов $\left(\mathrm{CH}_{3}\right)_{2} \mathrm{CO} \ldots \mathrm{HF}$ в области $3800-3200 \mathrm{~cm}^{-1}$ при $T=295 \mathrm{~K}$ и давлениях $P_{\mathrm{HF}}=10$ Torr, $P_{\text {acet }}=25$ Torr. A $\left({ }^{12} \mathrm{CH}_{3}\right){ }_{2}^{12} \mathrm{CO}, \mathrm{B}-\left({ }^{12} \mathrm{CH}_{3}\right){ }_{2}^{13} \mathrm{CO} . a-$ экспериментальный спектр поглощения смеси ацетон/НF, $b-$ экспериментальный спектр поглощения ацетона, $c-$ спектр поглощения комплекса. Стрелками отмечены частоты полос, представленных в табл. 1. 
Таблица 1. Экспериментальные значения частот $v /$ спектральных моментов $M_{1}\left(\mathrm{~cm}^{-1}\right)$ полос поглощения свободных молекул $\left({ }^{12} \mathrm{CH}_{3}\right){ }_{2}^{12} \mathrm{CO}$ и $\left({ }^{12} \mathrm{CH}_{3}\right){ }_{2}^{13} \mathrm{CO}$ и комплексов $\left({ }^{12} \mathrm{CH}_{3}\right){ }_{2}^{12} \mathrm{CO} \ldots \mathrm{HF}$ и $\left({ }^{12} \mathrm{CH}_{3}\right){ }_{2}^{13} \mathrm{CO} \ldots \mathrm{HF}$

\begin{tabular}{|c|c|c|c|c|}
\hline $\begin{array}{c}\text { Полоса } \\
\text { поглощения }\end{array}$ & $\begin{array}{c}\left({ }^{12} \mathrm{CH}_{3}\right)_{2}^{12} \mathrm{CO} \\
v / M_{1}\end{array}$ & $\begin{array}{c}\left({ }^{12} \mathrm{CH}_{3}\right)_{2}^{13} \mathrm{CO} \\
v / M_{1}\end{array}$ & $\begin{array}{c}\left({ }^{12} \mathrm{CH}_{3}\right)_{2}^{12} \mathrm{CO} \ldots \mathrm{HF} \\
v / M_{1}\end{array}$ & $\begin{array}{c}\left({ }^{12} \mathrm{CH}_{3}\right)_{2}^{13} \mathrm{CO} \ldots \mathrm{HF} \\
v / M_{1}\end{array}$ \\
\hline $\mathrm{H}-\mathrm{F}$ & - & - & $3460 / 3530(5)$ & $3457 / 3520(5)$ \\
\hline $2 v(\mathrm{C}=\mathrm{O})$ & $3455 / 3459(3)$ & $3382 / 3388(3)$ & - & - \\
\hline $\mathrm{C}-\mathrm{H}$ & $2970,3017 / 2987(2)$ & $2970,3016 / 2983(2)$ & - & - \\
\hline $\mathrm{C}=\mathrm{O}$ & $1740 / 1742(2)$ & $1700 / 1702(3)$ & $1722 / 1724(3)$ & $1681 / 1685(3)$ \\
\hline $\mathrm{H}-\mathrm{C}-\mathrm{H}$ & $1365 / 1395(2)$ & $1362 / 1396(2)$ & $1376 / 1418(2)$ & $1372 / 1386(2)$ \\
\hline $\mathrm{C}-\mathrm{C}$ & $1217 / 1216(2)$ & $1191 / 1190(2)$ & $1235 / 1235(2)$ & $1210 / 1208(2)$ \\
\hline $\mathrm{CH}_{3}$ & 1094,1091 & 1081,1079 & - & - \\
\hline
\end{tabular}

Примечание. Погрешность определения значений $M_{1}$ показана в круглых скобках.

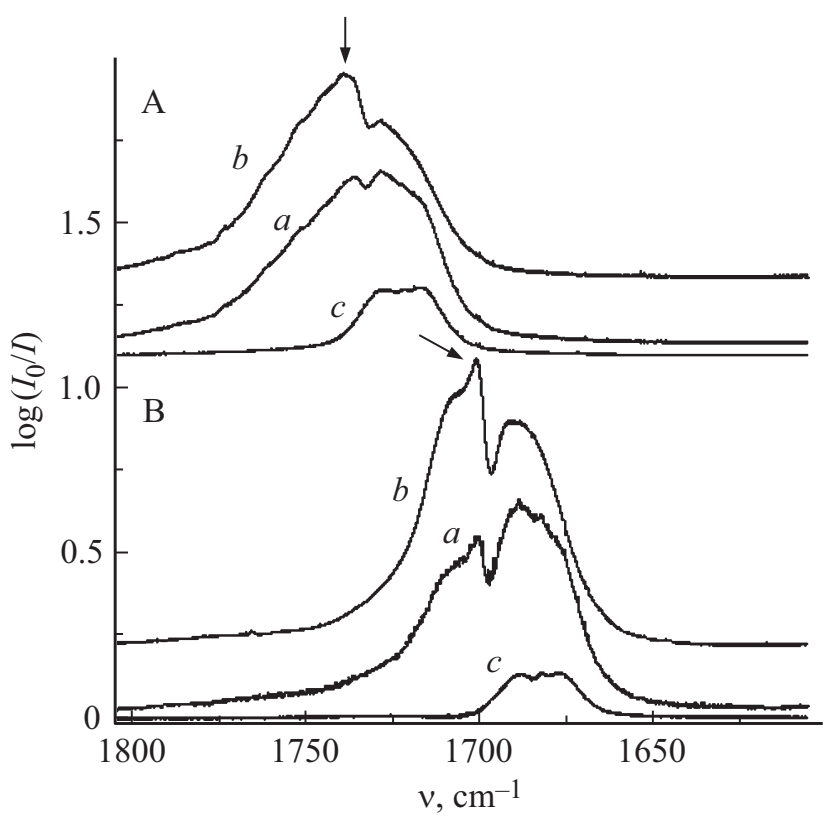

Pис. 3. Спектры поглощения свободных молекул $\left(\mathrm{CH}_{3}\right)_{2} \mathrm{CO}$ и комплексов $\left(\mathrm{CH}_{3}\right)_{2} \mathrm{CO} \ldots \mathrm{HF}$ в области частоты колебания $\mathrm{C}=\mathrm{O}$ при $T=295 \mathrm{~K}$ и давлениях $P_{\mathrm{HF}}=10$ Torr, $P_{\text {acet }}=25$ Torr. $\mathrm{A}-\left({ }^{12} \mathrm{CH}_{3}\right){ }_{2}^{12} \mathrm{CO}, \mathrm{B}-\left({ }^{12} \mathrm{CH}_{3}\right){ }_{2}^{13} \mathrm{CO} . a-$ экспериментальный спектр поглощения смеси ацетон/НF, $b-$ экспериментальный спектр поглощения ацетона, $c$ - спектр поглощения комплекса. Стрелками отмечены частоты полос, представленных в табл. 1.

\section{Расчет оптимальной геометрии и спектральных параметров молекулы $\left({ }^{12} \mathrm{CH}_{3}\right){ }_{2}^{13} \mathrm{CO}$ и комплекса $\left({ }^{12} \mathrm{CH}_{3}\right){ }_{2}^{13} \mathrm{CO} \ldots$. . HF}

Оптимальные ядерные конфигурации свободной молекулы ацетона и комплекса ацетон...НF были рассчитаны в приближении MP2/6-311++G(3df,3pd) с использованием пакета программ Gaussian 16 [10]. При расчете комплекса была учтена поправка на ошибку наложения базисных наборов мономеров. Оптимальная геометрия комплекса показана на рис. 5. Для

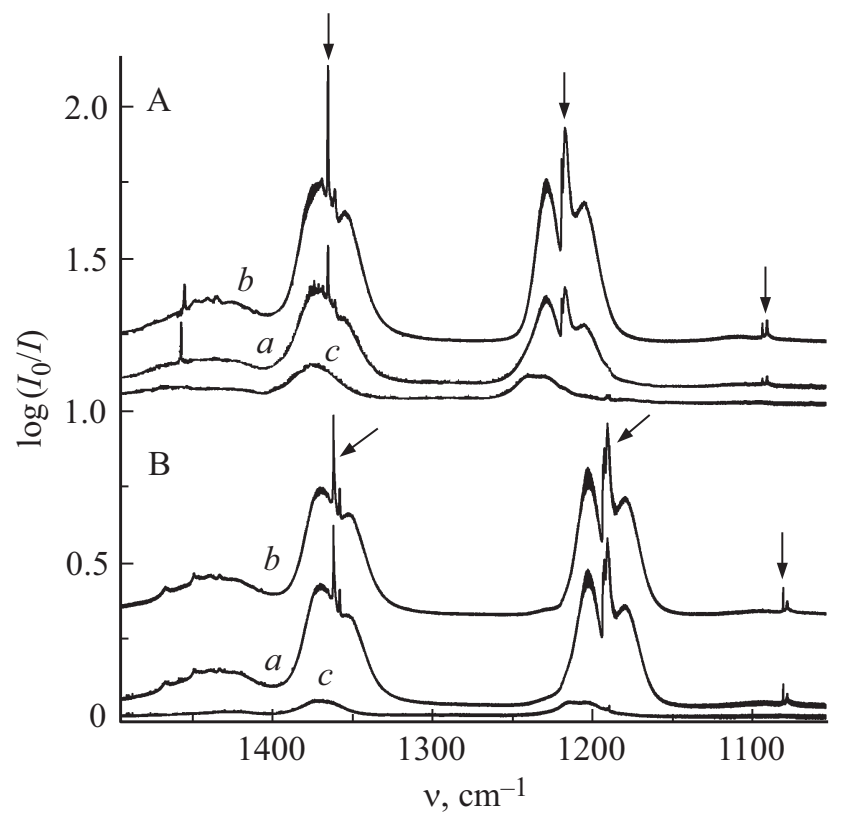

Pис. 4. Спектры поглощения свободных молекул $\left(\mathrm{CH}_{3}\right)_{2} \mathrm{CO}$ и комплексов $\left(\mathrm{CH}_{3}\right)_{2} \mathrm{CO} \ldots \mathrm{HF}$ в области $1450-1100 \mathrm{~cm}^{-1}$ при $T=295 \mathrm{~K}$ и давлениях $P_{\mathrm{HF}}=10$ Torr, $P_{\text {acet }}=25$ Torr. A $\left({ }^{12} \mathrm{CH}_{3}\right){ }_{2}^{12} \mathrm{CO}, \mathrm{B}-\left({ }^{12} \mathrm{CH}_{3}\right){ }_{2}^{13} \mathrm{CO} . \quad a-$ экспериментальный спектр поглощения смеси ацетон/HF, $b$ - экспериментальный спектр поглощения ацетона, $c$ - спектр поглощения комплекса. Стрелками отмечены частоты полос, представленных в табл. 1.

симметричной молекулы ацетона получены следующие значения геометрических параметров (для атомов свободной молекулы используются обозначения рис. 5): $r\left(\mathrm{C}_{1} \mathrm{O}\right)=1.2163, r\left(\mathrm{C}_{1} \mathrm{C}_{2}\right)=1.5089, r\left(\mathrm{C}_{2} \mathrm{H}_{1}\right)=$ $=1.0850, r\left(\mathrm{C}_{2} \mathrm{H}_{2}\right)=1.0901 \AA$; угол $\mathrm{OC}_{1} \mathrm{C}_{2}=121.89^{\circ}$, $\mathrm{C}_{2} \mathrm{C}_{1} \mathrm{C}_{3}=116.23^{\circ}, \mathrm{H}_{1} \mathrm{C}_{2} \mathrm{C}_{1}=110.11^{\circ}, \mathrm{H}_{3} \mathrm{C}_{2} \mathrm{C}_{1}=109.84^{\circ}$, $\mathrm{H}_{1} \mathrm{C}_{2} \mathrm{H}_{3}=109.95^{\circ}$. Оптимальные значения межьядерных расстояний и углов между связями комплекса приведены в табл. 2. Сравнение этих данных со значениями геометрических параметров, полученными ранее $[3,7]$ для $\left(\mathrm{CH}_{3}\right)_{2} \mathrm{CO} \ldots \mathrm{HF}$ с менее полным набором атомных функций 6-311++G(2d,2p), показало, что различия 

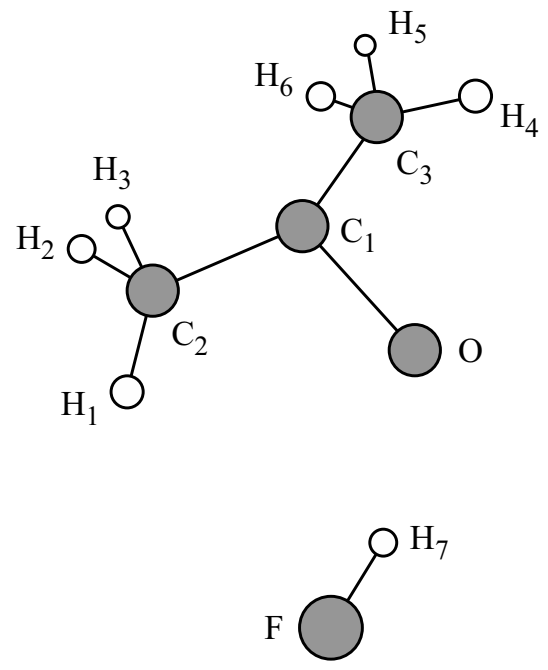

Рис. 5. Равновесная геометрия комплекса $\left(\mathrm{CH}_{3}\right)_{2} \mathrm{CO}$...HF.

между более точными значениями внутримолекулярных расстояний комплекса и значениями из $[3,7]$ не превышают $0.003 \AA$, а новое значения $R_{\mathrm{FO}}$ меньше прежнего на $0.0271 \AA$. Различия между двумя наборами значений внутримолекулярных углов не превышают $0.1^{\circ}$, а углы, характеризующие взаимную ориентацию мономеров в комплексе, отличаются не более чем на $0.3^{\circ}$. Значения дипольного момента комплекса, полученные с наборами атомных функций 6-311++G $(2 \mathrm{~d}, 2 \mathrm{p})$ и 6$311++\mathrm{G}(3 \mathrm{df}, 3 \mathrm{pd})$, равны 5.8154 и $5.7455 \mathrm{D}$. Близость энергий связи комплексов 9.20 и $9.68 \mathrm{kcal} \mathrm{mol}^{-1}$, полученных с использованием менее и более полных наборов атомных функций, также показывает, что набор $6-311++\mathrm{G}(2 \mathrm{~d}, 2 \mathrm{p})$ обеспечивает достаточно точное описание рассматриваемых систем.

В табл. 3 приведены гармонические и ангармонические значения частот и гармонические значения интенсивностей спектральных переходов в свободных молекулах $\left({ }^{12} \mathrm{CH}_{3}\right){ }_{2}^{12} \mathrm{CO}$ и $\left({ }^{12} \mathrm{CH}_{3}\right){ }_{2}^{13} \mathrm{CO}$, вычисленные в приближении MP2/6-311++G $(2 \mathrm{~d}, 2 \mathrm{p})$. Ангармонический расчет выполнен по методу теории возмущений [11-13] с учетом взаимодействия всех нормальных колебаний. Значения приведенных масс колебаний также указаны в табл. 3, так как они содержат информацию о типе колебания и могут меняться при изотопном замещении. Переходы указаны в порядке возрастания частоты, их нумерация соответствует нумерации нормальных колебаний в программе Gaussian. В табл. 3 использованы следующие обозначения типов колебаний: $\mathrm{C}-\mathrm{C}$ и $\mathrm{C}=\mathrm{O}-$ валентные колебания связей, $\delta(\mathrm{CCC})-$ внутренние деформационные колебания группы атомов, $\rho\left(\mathrm{CH}_{3}\right)-$ деформационные колебания группы атомов как целого в плоскости молекулы, $\omega\left(\mathrm{CH}_{3}\right)$ - деформационные колебания группы атомов как целого с выходом из плоскости симметрии. Из этой таблицы видно, что изменения частот спектральных переходов молекул ацетона при замещении ${ }^{12} \mathrm{C} \rightarrow{ }^{13} \mathrm{C}$ в карбонильной группе, определен- ные по гармоническим значениям частот, как правило, очень близки изменениям, вычисленным по ангармоническим частотам. Расчеты показывают, что наиболее интенсивными в спектре поглощения молекулярного ацетона должны быть фундаментальные переходы по колебаниям связи $\mathrm{C}=\mathrm{O}$ и антисимметричным валентным колебаниям связей $\mathrm{C}-\mathrm{C}$ и деформационным колебаниям НСН. При переходе от $\left({ }^{12} \mathrm{CH}_{3}\right){ }_{2}^{12} \mathrm{CO}$ к $\left({ }^{12} \mathrm{CH}_{3}\right){ }_{2}^{13} \mathrm{CO}$ частота наиболее интенсивной фундаментальной полосы $v_{18}\left(\mathrm{C}=\mathrm{O}, A_{1}\right)$ понижается, согласно ангармоническому расчету, на $39 \mathrm{~cm}^{-1}$, а частота первого обертона этой полосы понижается на $76 \mathrm{~cm}^{-1}$.

В табл. 4 представлены теоретические значения частот, интенсивностей и приведенных масс для наиболее интенсивных фундаментальных ИК полос поглощения комплексов $\left({ }^{12} \mathrm{CH}_{3}\right){ }_{2}^{12} \mathrm{CO} . . \mathrm{HF}$ и $\left({ }^{12} \mathrm{CH}_{3}\right){ }_{2}^{13} \mathrm{CO} . . \mathrm{HF}$. Нумерация колебаний взята из расчета нормальных колебаний в программе Gaussian. Обозначения типов колебаний такие же, как и в табл. $3, A^{\prime}$ и $A^{\prime \prime}-$ симметричное и антисимметричное неприводимое представление группы симметрии $C_{s}, l(\mathrm{HF}), A^{\prime}$ обозначает либрационное колебание мономера HF в плоскости комплекса, индексы $s$ и $a$ указывают приближенную симметричность или антисимметричность колебаний относительно перестановки групп $\mathrm{CH}_{3}$. Значения, отмеченные значком I, были получены с использованием набора атомных функций 6-311++G(2d,2p); значения, помеченные значком II, получены с более полным набором функций 6-311++G(3df, 3pd). Данные работ $[3,7]$ дополнены расчетом ангармонических частот $v_{\text {anh }}(\mathrm{I})$ комплекса $\left({ }^{12} \mathrm{CH}_{3}\right)_{2}^{12} \mathrm{CO} \ldots$... НF. Ангармонические значения, представленные в этой таблице, получены по теории возмущений с учетом взаимодействия всех мод. Использование наборов атомных функций I и II при расчете $\left({ }^{12} \mathrm{CH}_{3}\right){ }_{2}^{13} \mathrm{CO} \ldots$ HF приводит к очень близким значениям $v_{h}$ и $S_{h}$. Только для переходов $v_{9}\left(l(\mathrm{HF}), A^{\prime \prime}\right), v_{10}\left(l(\mathrm{HF}), A^{\prime}\right), v_{23}(\mathrm{C}=\mathrm{O})$ и $v_{30}(\mathrm{H}-\mathrm{F})$ расхождения между $v_{h}(\mathrm{I})$ и $v_{h}(\mathrm{II})$ могут достигать 20-30 $\mathrm{cm}^{-1}$. Ради экономии места значения $S_{h}(\mathrm{I})$ и $v_{\text {anh }}(\mathrm{I})$ для переходов $\left({ }^{12} \mathrm{CH}_{3}\right){ }_{2}^{13} \mathrm{CO} \ldots \mathrm{HF}$ в табл. 4 не указываются. Наиболее точными теоретическими значениями частот и интенсивностей комплекca $\left({ }^{12} \mathrm{CH}_{3}\right){ }_{2}^{13} \mathrm{CO} \ldots \mathrm{HF}$ являются ангармонические значения $v_{\text {anh }}(\mathrm{II})$ и $S_{\text {anh }}(\mathrm{II})$, которые будут сравниваться далее с экспериментальными данными. Анализ значений $v_{h}(\mathrm{I})$ и $v_{\mathrm{anh}}(\mathrm{I})$, полученных для $\left({ }^{12} \mathrm{CH}_{3}\right)_{2}^{12} \mathrm{CO} \ldots \mathrm{HF}$ и $\left({ }^{12} \mathrm{CH}_{3}\right){ }_{2}^{13} \mathrm{CO} \ldots$. . HF, показывает, что, как и в расчете свободных молекул ацетона, изменения частот спектральных переходов комплекса при замещении ${ }^{12} \mathrm{C} \rightarrow{ }^{13} \mathrm{C}$ в карбонильной группе, определенные по гармоническим значениям частот, очень близки изменениям, вычисленным по ангармоническим частотам. Исключением являются переходы, связанные с колебаниями, степень ангармонического взаимодействия которых с другими модами существенно меняется при изотопном замещении. Например, либрационное колебание $v_{10}\left(l(\mathrm{HF}), A^{\prime}\right)$ и валентное колебание $v_{11}(\mathrm{C}-\mathrm{C}, s)$ в гармоническом 
Таблица 2. Оптимальные структурные параметры комплекса ацетон. . .НF, вычисленные в приближении MP2/6-311++G(3df, 3pd)

\begin{tabular}{|c|c|c|c|c|c|c|c|c|}
\hline \multicolumn{9}{|c|}{ Расстояния, А } \\
\hline $\begin{array}{c}r_{\mathrm{C}_{1} \mathrm{O}} \\
1.2231\end{array}$ & $\begin{array}{c}r_{\mathrm{C}_{1} \mathrm{C}_{2}} \\
1.5027\end{array}$ & $\begin{array}{c}r_{\mathrm{C}_{1} \mathrm{C}_{3}} \\
1.5021\end{array}$ & $\begin{array}{c}r_{\mathrm{C}_{2} \mathrm{H}_{1}} \\
1.0850\end{array}$ & $\begin{array}{c}r_{\mathrm{C}_{2} \mathrm{H}_{2}} \\
1.0899\end{array}$ & $\begin{array}{c}r_{\mathrm{C}_{3} \mathrm{H}_{4}} \\
1.0847\end{array}$ & $\begin{array}{c}r_{\mathrm{C}_{3} \mathrm{H}_{5}} \\
1.0898\end{array}$ & $\begin{array}{c}R_{\mathrm{F} \ldots \mathrm{O}} \\
2.6304\end{array}$ & $\begin{array}{c}r_{\mathrm{FH}}, \\
0.9384\end{array}$ \\
\hline \multicolumn{9}{|c|}{ Углы, deg } \\
\hline $\begin{array}{l}\mathrm{OC}_{1} \mathrm{C}_{2} \\
122.21\end{array}$ & $\begin{array}{r}\mathrm{OC}_{1} \mathrm{C}_{3} \\
120.77\end{array}$ & $\begin{array}{c}\mathrm{H}_{1} \mathrm{C}_{2} \mathrm{C}_{1} \\
111.08\end{array}$ & $\begin{array}{c}\mathrm{H}_{3} \mathrm{C}_{2} \mathrm{C}_{1} \\
109.34\end{array}$ & $\begin{array}{c}\mathrm{FOC}_{1} \\
117.26\end{array}$ & $\begin{array}{c}\mathrm{C}_{1} \mathrm{C}_{3} \mathrm{H}_{4} \\
110.36\end{array}$ & $\begin{array}{c}\mathrm{C}_{1} \mathrm{C}_{3} \mathrm{H}_{5} \\
109.60\end{array}$ & $\begin{array}{c}\mathrm{OFH}_{7} \\
4.30\end{array}$ & $\begin{array}{r}\mathrm{C}_{1} \mathrm{OH}_{7} \\
119.76\end{array}$ \\
\hline
\end{tabular}

Таблица 3. Гармонические $\left(v_{h}\right)$ и ангармонические $\left(v_{\mathrm{anh}}\right)$ значения частот $\left(\mathrm{cm}^{-1}\right)$, приведенные массы $\mu$ (atomic units) и гармонические значения интенсивности $S_{h}\left(\mathrm{~km} \mathrm{~mol}^{-1}\right)$ для наиболее интенсивных фундаментальных полос поглощения и обертона $2 v(\mathrm{C}=\mathrm{O})$, вычисленные для молекул $\left({ }^{12} \mathrm{CH}_{3}\right){ }_{2}^{12} \mathrm{CO}$ и $\left({ }^{12} \mathrm{CH}_{3}\right){ }_{2}^{13} \mathrm{CO}$ с набором атомных функций 6-311++ $\mathrm{G}(2 \mathrm{~d}, 2 \mathrm{p})$

\begin{tabular}{|c|c|c|c|c|c|c|c|c|}
\hline \multirow{2}{*}{ Переход } & \multicolumn{4}{|c|}{$\left({ }^{12} \mathrm{CH}_{3}\right){ }_{2}^{12} \mathrm{CO}$} & \multicolumn{4}{|c|}{$\left({ }^{12} \mathrm{CH}_{3}\right){ }_{2}^{13} \mathrm{CO}$} \\
\hline & $v_{h}$ & $S_{h}$ & $\mu$ & $v_{\text {anh }}$ & $v_{h}$ & $S_{h}$ & $\mu$ & $v_{\text {anh }}$ \\
\hline$\nu_{3}\left(\delta(\mathrm{CCC}), A_{1}\right)$ & 380 & 1.3 & 2.1873 & 377 & 378 & 1.2 & 2.1936 & 375 \\
\hline$\nu_{5}\left(\delta(\mathrm{OCC}), B_{2}\right)$ & 533 & 14 & 3.6068 & 530 & 531 & 14 & 3.6359 & 527 \\
\hline$v_{6}\left((\mathrm{C}-\mathrm{C}), A_{1}\right)$ & 799 & 1.8 & 3.6460 & 776 & 797 & 2.0 & 3.6558 & 775 \\
\hline$\nu_{8}\left(\rho\left(\mathrm{CH}_{3}\right), B_{2}\right)$ & 903 & 6.6 & 1.5246 & 905 & 900 & 7.4 & 1.5612 & 901 \\
\hline$v_{10}\left(\omega\left(\mathrm{CH}_{3}\right), B_{1}\right)$ & 1129 & 1.4 & 1.9279 & 1095 & 1115 & 1.2 & 1.8754 & 1082 \\
\hline$v_{11}\left(\mathrm{C}-\mathrm{C}, B_{2}\right)$ & 1255 & 60 & 2.6569 & 1219 & 1227 & 60 & 2.7975 & 1193 \\
\hline$v_{12}\left(\delta(\mathrm{HCH}), A_{1}\right)$ & 1408 & 15 & 1.2354 & 1367 & 1407 & 16 & 1.2354 & 1367 \\
\hline$v_{13}\left(\delta(\mathrm{HCH}), B_{2}\right)$ & 1412 & 57 & 1.4822 & 1374 & 1408 & 49 & 1.4138 & 1371 \\
\hline$v_{14}\left(\delta(\mathrm{HCH}), B_{2}\right)$ & 1487 & 0.4 & 1.0417 & 1455 & 1487 & 0.6 & 1.0415 & 1454 \\
\hline$v_{16}\left(\delta(\mathrm{HCH}), A_{1}\right)$ & 1495 & 32 & 1.0763 & 1459 & 1494 & 34 & 1.0860 & 1456 \\
\hline$v_{17}\left(\delta(\mathrm{HCH}), B_{1}\right)$ & 1515 & 20 & 1.0445 & 1460 & 1514 & 20 & 1.0438 & 1470 \\
\hline$v_{18}\left(\mathrm{C}=\mathrm{O}, A_{1}\right)$ & 1746 & 128 & 8.4903 & 1709 & 1704 & 118 & 8.1855 & 1670 \\
\hline$v_{19}\left(\mathrm{C}-\mathrm{H}, B_{2}\right)$ & 3079 & 1.0 & 1.0363 & 2975 & 3079 & 1.1 & 1.0363 & 2973 \\
\hline$v_{20}\left(\mathrm{C}-\mathrm{H}, A_{1}\right)$ & 3084 & 5.9 & 1.0361 & 2971 & 3084 & 5.9 & 1.0361 & 2971 \\
\hline$v_{22}\left(\mathrm{C}-\mathrm{H}, B_{1}\right)$ & 3161 & 12 & 1.0993 & 3017 & 3161 & 12 & 1.0993 & 3017 \\
\hline$v_{23}\left(\mathrm{C}-\mathrm{H}, B_{2}\right)$ & 3205 & 8 & 1.1032 & 3062 & 3205 & 8 & 1.1032 & 3062 \\
\hline$v_{24}\left(\mathrm{C}-\mathrm{H}, A_{1}\right)$ & 3206 & 3.7 & 1.1035 & 3062 & 3206 & 3.6 & 1.1035 & 3064 \\
\hline $2 v_{18}\left(\mathrm{C}=\mathrm{O}, A_{1}\right)$ & 3493 & - & 8.4903 & 3400 & 3408 & - & 8.1855 & 3324 \\
\hline
\end{tabular}

Примечание. Полоса $\left({ }^{12} \mathrm{CH}_{3}\right){ }_{2}^{13} \mathrm{CO}$ при $v_{h}=900 \mathrm{~cm}^{-1}$ имеет номер 7.

приближении слабо взаимодействуют друг с другом в комплексе с ${ }^{12} \mathrm{C}$ и очень сильно в комплексе с ${ }^{13} \mathrm{C}$. В результате колебание $v_{11}(\mathrm{C}-\mathrm{C}, s)$ комплекca $\left({ }^{12} \mathrm{CH}_{3}\right){ }_{2}^{13} \mathrm{CO} \ldots$... зF заимствует значительную часть интенсивности либрационного перехода: $S_{h}(\mathrm{II})=117$ и $52 \mathrm{~km} \mathrm{~mol}^{-1}$ для $v_{10}\left(l(\mathrm{HF}), A^{\prime}\right)$ и $v_{11}(\mathrm{C}-\mathrm{C}, s)$ соответственно. Но учет ангармонического взаимодействия колебаний $v_{10}$ и $v_{11}$ друг с другом и с остальными модами приводит к значениям интенсивности 157 и $1.9 \mathrm{~km} \mathrm{~mol}^{-1}$ для $v_{10}$ и $v_{11}$, которые близки к значениям $S_{h}(\mathrm{I})$ для $\left({ }^{12} \mathrm{CH}_{3}\right){ }_{2}^{12} \mathrm{CO} \ldots \mathrm{HF}$. Значения $v_{h}(\mathrm{I})$ для перехода $v_{30}(\mathrm{H}-\mathrm{F})$, вычисленные для двух изотопологов комплекса, практически совпадают. То же самое верно и для значений $v_{\mathrm{anh}}(\mathrm{I})$. Это позволяет сделать вывод, что расчеты обоих изотопологов с базисным набором 6-311++G(3df,3pd) тоже должны давать одно и то же значение $v_{\text {anh }}($ II). Следовательно, полосе $v_{30}(\mathrm{H}-\mathrm{F})$ более легкого изотополога мы мо- жем приписать более точное значение $v_{\text {anh }}(\mathrm{II})$, равное $3489 \mathrm{~cm}^{-1}$. Замечая, что разность значений $v_{h}(\mathrm{I})$ для полосы $v_{23}(\mathrm{C}=\mathrm{O})$ двух изотопологов $\left(42 \mathrm{~cm}^{-1}\right)$ почти совпадает с аналогичной разностью значений $v_{\text {anh }}(\mathrm{I})$ $\left(41 \mathrm{~cm}^{-1}\right)$, и используя значение $v_{\mathrm{anh}}(\mathrm{II})=1686 \mathrm{~cm}^{-1}$ для $\left({ }^{12} \mathrm{CH}_{3}\right){ }_{2}^{13} \mathrm{CO} \ldots \mathrm{HF}$, можно получить оценку для значения $v_{\mathrm{anh}}(\mathrm{II})$ в $\left({ }^{12} \mathrm{CH}_{3}\right){ }_{2}^{12} \mathrm{CO} \ldots \mathrm{HF}$, равную $1727 \mathrm{~cm}^{-1}$.

Ангармонический расчет по теории возмущений второго порядка [11-13], выполненный для комплекса $\left({ }^{12} \mathrm{CH}_{3}\right){ }_{2}^{13} \mathrm{CO} \ldots \mathrm{HF}$, предсказывает, что в исследуемой области спектра помимо полос фундаментальных переходов могут наблюдаться достаточно интенсивные полосы обертонных и составных переходов. В табл. 5 показаны частоты и интенсивности таких переходов, интенсивности которых не ниже $1 \mathrm{~km} \mathrm{~mol}^{-1}$. Обозначения большей части колебаний пояснены в табл. 4. Остальные колебания имеют следующий смысл: $v_{2}$ деформационное колебание мономера ацетона с выхо- 
Таблица 4. Частоты $v\left(\mathrm{~cm}^{-1}\right)$, интенсивности $S\left(\mathrm{~km} \mathrm{~mol}^{-1}\right)$ и приведенные массы $\mu$ (atomic units) для наиболее интенсивных фундаментальных полос поглощения комплексов $\left({ }^{12} \mathrm{CH}_{3}\right){ }_{2}^{12} \mathrm{CO} \ldots \mathrm{HF}$ и $\left({ }^{12} \mathrm{CH}_{3}\right){ }_{2}^{13} \mathrm{CO} \ldots \mathrm{HF}$

\begin{tabular}{|c|c|c|c|c|c|c|c|c|c|c|}
\hline \multirow{2}{*}{ Переход } & \multicolumn{4}{|c|}{$\left({ }^{12} \mathrm{CH}_{3}\right)_{2}^{12} \mathrm{CO} \ldots \mathrm{HF}$} & \multicolumn{6}{|c|}{$\left({ }^{12} \mathrm{CH}_{3}\right){ }_{2}^{13} \mathrm{CO} \ldots \mathrm{HF}$} \\
\hline & $v_{h}(\mathrm{I})$ & $S_{h}(\mathrm{I})$ & $\mu$ & $v_{\text {anh }}(\mathrm{I})$ & $v_{h}(\mathrm{I})$ & $v_{h}(\mathrm{II})$ & $S_{h}(\mathrm{II})$ & $\mu$ & $v_{\text {anh }}($ II $)$ & $S_{\text {anh }}($ II $)$ \\
\hline$v_{3}\left(\delta(\right.$ acet $\left.), A^{\prime}\right)$ & 77 & 4.4 & 5.87 & 65 & 77 & 82 & 4.4 & 5.92 & 71 & 3.3 \\
\hline$v_{5}(\mathrm{O} \ldots \mathrm{H})$ & 199 & 10 & 6.64 & 181 & 199 & 205 & 10.1 & 6.59 & 181 & 6.4 \\
\hline$\nu_{6}(\delta(\mathrm{CCC}))$ & 391 & 3.4 & 2.22 & 389 & 390 & 391 & 3.4 & 2.22 & 384 & 2.6 \\
\hline$v_{8}(\delta(\mathrm{OCC}))$ & 555 & 28 & 3.64 & 546 & 553 & 556 & 29 & 3.66 & 546 & \\
\hline $25 v_{9}\left(l(\mathrm{HF}), A^{\prime \prime}\right)$ & 755 & 105 & 1.04 & 671 & 755 & 777 & 93 & 1.05 & 694 & 95 \\
\hline$v_{10}\left(l(\mathrm{HF}), A^{\prime}\right)$ & 791 & 169 & 1.07 & 691 & 791 & 814 & 117 & 1.43 & 694 & 157 \\
\hline$v_{11}(\mathrm{C}-\mathrm{C}, s)$ & 814 & 1.4 & 3.48 & 795 & 812 & 822 & 52 & 1.88 & 821 & 71.9 \\
\hline$v_{13}\left(\delta\left(\mathrm{CH}_{3}\right), a, A^{\prime}\right)$ & 918 & 3.8 & 1.50 & 913 & 915 & 913 & 3.3 & 1.53 & 917 & 7.9 \\
\hline$v_{15}\left(\omega\left(\mathrm{CH}_{3}\right), s, A^{\prime \prime}\right)$ & 1135 & 2.7 & 1.94 & 1102 & 1121 & 1114 & 3.1 & 1.91 & 1073 & 4.3 \\
\hline$v_{16}(\mathrm{C}-\mathrm{C}, a)$ & 1279 & 54 & 2.58 & 1241 & 1251 & 1252 & 52 & 2.61 & 1212 & 57 \\
\hline$v_{17}\left(\delta(\mathrm{HCH}), s, A^{\prime}\right)$ & 1416 & 23 & 1.26 & 1376 & 1415 & 1403 & 24 & 1.26 & 1360 & 15 \\
\hline$v_{18}\left(\delta(\mathrm{HCH}), a, A^{\prime}\right)$ & 1423 & 53 & 1.46 & 1384 & 1420 & 1410 & 50 & 1.44 & 1368 & 52 \\
\hline$v_{19}\left(\delta(\mathrm{HCH}), a, A^{\prime}\right)$ & 1487 & 2.8 & 1.04 & 1453 & 1486 & 1474 & 3.6 & 1.04 & 1440 & 1.9 \\
\hline$v_{21}\left(\delta(\mathrm{HCH}), s, A^{\prime}\right)$ & 1495 & 39 & 1.09 & 1442 & 1494 & 1485 & 37 & 1.09 & 1422 & 26 \\
\hline$v_{22}\left(\delta(\mathrm{HCH}), s, A^{\prime \prime}\right)$ & 1516 & 22 & 1.04 & 1466 & 1515 & 1508 & 22 & 1.04 & 1447 & 7.2 \\
\hline$v_{23}(\mathrm{C}=\mathrm{O})$ & 1736 & 173 & 7.83 & 1704 & 1694 & 1715 & 170 & 8.17 & 1686 & 123 \\
\hline$v_{25}\left(\mathrm{C}-\mathrm{H}, s, A^{\prime}\right)$ & 3088 & 4.0 & 1.04 & 2963 & 3088 & 3076 & 3.6 & 1.04 & 2993 & 1.8 \\
\hline$v_{27}\left(\mathrm{C}-\mathrm{H}, s, A^{\prime \prime}\right)$ & 3164 & 5.9 & 1.10 & 3025 & 3164 & 3159 & 4.2 & 1.10 & 3052 & 2.5 \\
\hline$v_{28}\left(\mathrm{C}-\mathrm{H}, a, A^{\prime}\right)$ & 3210 & 2.7 & 1.10 & 3075 & 3210 & 3206 & 1.4 & 1.10 & 3082 & 2.0 \\
\hline$v_{29}\left(\mathrm{C}-\mathrm{H}, s, A^{\prime}\right)$ & 3211 & 1.8 & 1.10 & 3059 & 3211 & 3207 & 2.3 & 1.10 & 3064 & 2.6 \\
\hline$v_{30}(\mathrm{H}-\mathrm{F})$ & 3712 & 1147 & 1.06 & 3533 & 3713 & 3680 & 1167 & 1.06 & 3489 & 887 \\
\hline
\end{tabular}

Таблица 5. Ангармонические значения частот $\left(\mathrm{cm}^{-1}\right)$ и интенсивностей $\left(\mathrm{km} \mathrm{mol}^{-1}\right)$ наиболее интенсивных обертонных и составных полос поглощения комплекса $\left({ }^{12} \mathrm{CH}_{3}\right){ }_{2}^{13} \mathrm{CO}$. .HF в исследуемой области спектра

\begin{tabular}{l|c|c|c|c|c|c|c|c}
\hline Переход & $\nu_{\text {anh }}(\mathrm{II})$ & $S_{\text {anh }}(\mathrm{II})$ & Переход & $v_{\text {anh }}(\mathrm{II})$ & $S_{\text {anh }}(\mathrm{II})$ & Переход & $\nu_{\text {anh }}(\mathrm{II})$ & $S_{\text {anh }}(\mathrm{II})$ \\
\hline$\nu_{2}+v_{9}$ & 767 & 1.7 & $v_{6}+v_{14}$ & 1451 & 4.0 & $2 v_{12}$ & 1740 & 1.0 \\
$\nu_{3}+v_{10}$ & 812 & 7.8 & $v_{8}+v_{13}$ & 1464 & 2.6 & $v_{8}+v_{16}$ & 1755 & 1.3 \\
$\nu_{3}+v_{11}$ & 838 & 4.3 & $v_{6}+v_{15}$ & 1466 & 5.9 & $2 v_{13}$ & 1827 & 2.7 \\
$\nu_{5}+v_{9}$ & 860 & 1.9 & $v_{10}+v_{11}$ & 1469 & 22 & $2 v_{23}$ & 3356 & 4.7 \\
$\nu_{5}+v_{10}$ & 918 & 2.4 & $2 v_{11}$ & 1531 & 5.1 & $v_{2}+v_{30}$ & 3571 & 1.5 \\
$2 v_{9}$ & 1296 & 72 & $v_{9}+v_{12}$ & 1556 & 4.8 & $v_{3}+v_{30}$ & 3572 & 86 \\
$\nu_{8}+v_{11}$ & 1313 & 2.2 & $v_{4}+v_{22}$ & 1563 & 1.1 & $v_{5}+v_{30}$ & 3696 & 49 \\
$2 v_{10}$ & 1449 & 21 & $v_{11}+v_{13}$ & 1684 & 1.1 & & &
\end{tabular}

дом из плоскости, $v_{4}$ - крутильное колебание метильной группы $\mathrm{CH}_{3}$ вокруг связи $\mathrm{C}-\mathrm{C}, v_{7}-$ колебание по двугранному углу ССОС, $v_{12}$ - деформационное внутреннее колебание $\mathrm{CH}_{3}$ и $v_{14}$ - качание группы $\mathrm{CH}_{3}$ перпендикулярно плоскости комплекса. Отметим, что расчет по теории возмущений [11-13] предсказывает появление еще нескольких интенсивных суммарных переходов, включающих деформационное колебание $v_{1}$, гармоническая частота которого равна $21 \mathrm{~cm}^{-1}$. Эти данные не приведены в табл. 5. В этом случае теория возмушений второго порядка приводит к явно ошибочным результатам, потому что величина эффектов ангармонического взаимодействия этого колебания с другими намного больше его частоты.
Чтобы изучить влияние внутримодовой ангармоничности и межмодовых взаимодействий на спектральные параметры, мы использовали вариационный метод решения колебательных уравнений Шредингера. В [14] было показано, что при расчете комплексов с водородной связью метод теории возмущений и вариационный метод могут удачно дополнять друг друга. В рассматриваемом комплексе наибольший интерес представляют валентные колебания связей $\mathrm{H}-\mathrm{F}$ и ${ }^{13} \mathrm{C}=\mathrm{O}$ и их взаимодействие с низкочастотным валентным и деформационными колебаниями водородной связи. При использовании вариационного метода в таких задачах важно выбрать оптимальный вид колебательных координат. В качестве таких координат можно брать изменения отдельных длин связей 
и углов [15] или нормальные координаты комплекса [16]. В [2] было показано, что выбор массово-взвешенных нормальных координат в качестве колебательных переменных предпочтителен при изучении изотопных эффектов, так как эффекты изменения масс ядер уже частично отражаются в форме нормальных координат. Детали этого варианта вариационного метода изложены в [16,17]. Для комплекса $\left({ }^{12} \mathrm{CH}_{3}\right){ }_{2}^{13} \mathrm{CO} \ldots$. HF вариационный метод был применен при рассмотрении одномерных ангармонических валентных колебаний связей $\mathrm{H}-\mathrm{F},{ }^{13} \mathrm{C}=\mathrm{O}$ и Н-связи и либрационных колебаний мономеров в плоскости комплекса, а также при решении двумерных задач для всех пар указанных колебаний. Для каждой степени свободы оператор кинетической энергии выражался через вторую производную колебательной координаты, и десять функций гармонического осциллятора использовались как базисные функции. Как и в [7], поверхности потенциальной энергии и дипольного момента рассчитывались с набором атомных функций 6-311++G(2d,2p). Расчеты показали, что колебание ${ }^{13} \mathrm{C}=\mathrm{O}$ обладает малой механической ангармоничностью $\omega_{e} x_{e}\left(4.5 \mathrm{~cm}^{-1}\right.$ в свободной молекуле $\left({ }^{12} \mathrm{CH}_{3}\right){ }_{2}^{13} \mathrm{CO}$ и $4.9 \mathrm{~cm}^{-1}$ в комплексе), в то время как ангармоничность колебания $\mathrm{H}-\mathrm{F}$ более значительна $\left(86 \mathrm{~cm}^{-1}\right)$ и возрастает до $137 \mathrm{~cm}^{-1}$ при образовании комплекса $\left({ }^{12} \mathrm{CH}_{3}\right){ }_{2}^{13} \mathrm{CO}$... HF. Напротив, электрооптическая ангармоничность колебания ${ }^{13} \mathrm{C}=\mathrm{O}$ в свободной молекуле ацетона и комплексе намного больше, чем у колебания $\mathrm{H}-\mathrm{F}$. Теоретические значения частоты основного тона и первого обертона колебания $\mathrm{C}=\mathrm{O}$ в комплексе изменяются менее чем на $1 \mathrm{~cm}^{-1}$ под действием ангармонического взаимодействия с межмолекулярными низкочастотными модами. Изменения интенсивности этих переходов также незначительны. Как и в комплексе $\left({ }^{12} \mathrm{CH}_{3}\right){ }_{2}^{12} \mathrm{CO} \ldots \mathrm{HF}$ [7], колебание $\mathrm{C}=\mathrm{O}$ испытывает более значительное возмущение со стороны валентного колебания $\mathrm{H}-\mathrm{F}$. Ангармоническая частота основного тона ${ }^{13} \mathrm{C}=\mathrm{O}$ понижается от 1686 до $1684 \mathrm{~cm}^{-1}$, а частота обертона понижается от 3365 до $3360 \mathrm{~cm}^{-1}$ при переходе от одномерного к двумерному приближению. При этом частота основного тона колебания $\mathrm{H}-\mathrm{F}$ тоже уменьшается от 3436 до $3432 \mathrm{~cm}^{-1}$. В комплексе $\left({ }^{12} \mathrm{CH}_{3}\right){ }_{2}^{12} \mathrm{CO}$. . HF одномерное значение частоты обертона $\mathrm{C}=\mathrm{O}$ было выше на $19 \mathrm{~cm}^{-1}$, чем одномерное значение частоты колебания $\mathrm{H}-\mathrm{F}$. Малое значение разности этих частот приводило к сильному $2 v(\mathrm{C}=\mathrm{O}) / v(\mathrm{H}-\mathrm{F})$ резонансу. В результате интенсивность обертона возрастала в 14 раз по сравнению с одномерным значением. В комплексе с ${ }^{13} \mathrm{C}=\mathrm{O}$ одномерное значение частоты обертона ниже на $71 \mathrm{~cm}^{-1}$, чем одномерное значение частоты колебания $\mathrm{H}-\mathrm{F}$. Изза большой величины этой разницы в более тяжелом комплексе примешивание волновой функции первого возбужденного колебания $\mathrm{H}-\mathrm{F}$ к волновой функции дважды возбужденного колебания $\mathrm{C}=\mathrm{O}$ менее значительно, и интенсивность обертона возрастает только в три раза.

\section{Обсуждение результатов эксперимента и расчета}

Реконструкция экспериментально наблюдаемых полос поглощения свободных молекул ацетона и комплексов ацетона с фтористым водородом в виде суперпозиции большого числа фундаментальных, составных и горячих переходов между колебательными состояниями, как это было сделано, например, в [18] для комплекса $\left(\mathrm{CH}_{3}\right)_{2} \mathrm{O}$. . HF, не являлась целью данной работы. Подобная реконструкция представляет самостоятельную и очень сложную задачу, тем более что молекула ацетона и ее комплекс с HF являются асимметричными волчками: теоретические значения вращательных постоянных $\left(\mathrm{B} \mathrm{cm}^{-1}\right)$ равны $A=0.3370, B=0.2860, C=0.1640$ для $\left({ }^{12} \mathrm{CH}_{3}\right){ }_{2}^{13} \mathrm{CO}$ и $A=0.2947, B=0.0728, C=0.0596$ для $\left({ }^{12} \mathrm{CH}_{3}\right){ }_{2}^{13} \mathrm{CO}$. . . НF. Следовательно, строгая классификация деталей каждой полосы не может быть выполнена. Поэтому в табл. 1 в качестве частоты наблюдаемой полосы поглощения приводится частота ее основного максимума. Сопоставление экспериментально наблюдаемых полос типам колебаний ядер осуществлялось с использованием теоретических данных табл. 3 и 4.

Из сравнения спектров молекул ацетона, содержащих ${ }^{12} \mathrm{CO}$ и ${ }^{13} \mathrm{CO}$, видно, что при замещении ${ }^{12} \mathrm{C} \rightarrow{ }^{13} \mathrm{C}$ уменьшаются частоты следующих полос: $2 v(\mathrm{C}=\mathrm{O})$ на $73, v(\mathrm{C}=\mathrm{O})$ на $40, v(\mathrm{C}-\mathrm{C})$ на 26 и неплоского колебания метильных групп $\omega\left(\mathrm{CH}_{3}\right)$ на $13 \mathrm{~cm}^{-1}$. Практически неизменными по положению и форме остаются полосы внутренних деформационных $\left(1450-1325 \mathrm{~cm}^{-1}\right)$ и валентных (в области $3000 \mathrm{~cm}^{-1}$ ) CН-колебаний. Важно, что соответствующие изменения спектральных моментов $M_{1}$ имеют те же знаки и почти те же абсолютные значения. В спектре комплекса при замещении ${ }^{12} \mathrm{C} \rightarrow{ }^{13} \mathrm{C}$ частоты фундаментальных переходов по колебаниям $v(\mathrm{C}=\mathrm{O})$ и $v(\mathrm{C}-\mathrm{C})$ понижаются на 41 и $25 \mathrm{~cm}^{-1}$ соответственно. При образовании комплекса частота полосы $v(\mathrm{C}=\mathrm{O})$ понижается на $18 \mathrm{~cm}^{-1}$, а частоты полос $\mathrm{H}-\mathrm{C}-\mathrm{H}$ и $\mathrm{C}-\mathrm{C}$ повышаются соответственно на 11 и $18 \mathrm{~cm}^{-1}$. В комплексе с ${ }^{13} \mathrm{CO}$ полоса обертона $2 v\left({ }^{13} \mathrm{C}=\mathrm{O}\right)$ выходит из-под центра полосы колебания $\mathrm{H}-\mathrm{F}$ и хорошо видна на ее низкочастотном крыле в экспериментальном спектре. Контуры сложных полос $v(\mathrm{H}-\mathrm{F})$ комплексов $\left({ }^{12} \mathrm{CH}_{3}\right)_{2}^{12} \mathrm{CO} \ldots \mathrm{HF}$ и $\left({ }^{12} \mathrm{CH}_{3}\right)_{2}^{13} \mathrm{CO} \ldots \mathrm{HF}$, полученные в результате вычитания поглощения свободных молекул, практически не отличаются друг от друга, а их центры тяжести в пределах ошибки измерений совпадают. При замещении ${ }^{12} \mathrm{C} \rightarrow{ }^{13} \mathrm{C}$ поведение полос комплекса, связанных с внутренними колебаниями мономера ацетона в комплексе, аналогично поведению указанных выше полос свободных молекул ацетона.

Выполненные расчеты частот спектральных переходов свободных молекул ацетона и комплексов ацетон. ..НF достаточно точны, и их результаты могут быть использованы для интерпретации наблюдаемых спектров. Например, расчет предсказывает значение $3489 \mathrm{~cm}^{-1}$ 
для частоты фундаментального перехода по валентному колебанию $\mathrm{H}-\mathrm{F}$ в комплексе $\left({ }^{12} \mathrm{CH}_{3}\right)_{2}^{13} \mathrm{CO}$. . НF. Это значение близко к частоте максимума наблюдаемой полосы $3457 \mathrm{~cm}^{-1}$ и почти совпадает с полусуммой частот максимума и первого спектрального момента. Как и эксперимент, расчет показывает, что эта частота практически не меняется при замещении ${ }^{12} \mathrm{C}=\mathrm{O} \rightarrow{ }^{13} \mathrm{C}=\mathrm{O}$. Разность между теоретическим и экспериментальным значениями частоты колебания ${ }^{13} \mathrm{C}=\mathrm{O}$ комплекса не более $5 \mathrm{~cm}^{-1}$. Предсказываемое экспериментом понижение на $41 \mathrm{~cm}^{-1}$ частоты $\mathrm{C}=\mathrm{O}$ комплекса при замещении ${ }^{12} \mathrm{C} \rightarrow{ }^{13} \mathrm{C}$ точно воспроизводится расчетом. Близость теоретических значений частот к экспериментальным данным для других полос комплекса и корреляция между абсолютными теоретическими и относительными экспериментальными значениями интенсивностей свидетельствуют о надежности классификации спектральных переходов, используемой в табл. 3-5. Расчеты показали, какие переходы дают вклады в наблюдаемые полосы поглощения. Например, в полосу деформационных колебаний $\mathrm{H}-\mathrm{C}-\mathrm{H}$ комплекса $\left({ }^{12} \mathrm{CH}_{3}\right){ }_{2}^{13} \mathrm{CO}$. . HF в области $1400 \mathrm{~cm}^{-1}$ вносят вклад 5 деформационных колебаний метильных групп (табл. 4), обертон $v_{10}\left(l(\mathrm{HF}), A^{\prime}\right)$ и четыре суммарных перехода (табл. 5). Все эти переходы должны быть учтены при реконструкции контура наблюдаемой полосы. Большая интенсивность обертонов колебаний $v_{9}\left(l(\mathrm{HF}), A^{\prime \prime}\right)$ и $v_{10}\left(l(\mathrm{HF}), A^{\prime}\right)$ подтверждается вариационным расчетом, который предсказал сильное ангармоническое взаимодействие этих либрационных колебаний с валентным колебанием $\mathrm{H}-\mathrm{F}$. Расчет по теории возмущений подтверждает важность учета вкладов суммарных переходов при реконструкции экспериментального спектра $\left({ }^{12} \mathrm{CH}_{3}\right){ }_{2}^{13} \mathrm{CO} \ldots$ HF в области валентного колебания $\mathrm{H}-\mathrm{F}$ (табл. 5). В свою очередь, вариационный расчет показал, что низкая интенсивность обертонного перехода $2 v\left({ }^{13} \mathrm{C}=\mathrm{O}\right)\left(4.7 \mathrm{~km} \mathrm{~mol}^{-1}\right)$, расположенного на низкочастотном крыле фундаментальной полосы $\mathrm{H}-\mathrm{F}$, объясняется слабостью резонанса $2 v\left({ }^{13} \mathrm{C}=\mathrm{O}\right) / v(\mathrm{H}-\mathrm{F})$.

\section{Заключение}

Спектры поглощения смесей $\left({ }^{12} \mathrm{CH}_{3}\right){ }_{2}^{13} \mathrm{CO} / \mathrm{HF}$ и свободных молекул $\left({ }^{12} \mathrm{CH}_{3}\right){ }_{2}^{13} \mathrm{CO}$ зарегистрированы в области $4000-800 \mathrm{~cm}^{-1}$ с использованием вакуумного фурье-спектрометра Bruker IFS-125 HR при комнатной температуре с разрешением $0.05 \mathrm{~cm}^{-1}$. Полосы поглощения комплекса $\left({ }^{12} \mathrm{CH}_{3}\right){ }_{2}^{13} \mathrm{CO} \ldots$. . НF получены вычитанием поглощения свободных молекул НF, ацетона и атмосферной воды из экспериментального спектра смесей. Оптимальный выбор экспериментальных условий и методики обработки спектров позволил надежно выделить из наблюдаемых спектров четыре полосы комплекса $\left({ }^{12} \mathrm{CH}_{3}\right)_{2}^{13} \mathrm{CO} \ldots$. . Н. Для полного изучения влияния изотопных эффектов на спектральные параметры спектры $\left({ }^{12} \mathrm{CH}_{3}\right){ }_{2}^{12} \mathrm{CO} \ldots \mathrm{HF}$ были дополнительно зарегистрированы в более широком диапазоне частот, чем это было сделано в [7]. Экспериментальные данные позволили изучить тенденции в сдвигах полос поглощения при изотопном замещении ${ }^{12} \mathrm{C} \rightarrow{ }^{13} \mathrm{C}$ в карбонильной группе и при образовании комплекса. Частоты и интенсивности полос поглощения комплексов $\left({ }^{12} \mathrm{CH}_{3}\right){ }_{2}^{12} \mathrm{CO} \ldots \mathrm{HF}$ и $\left({ }^{12} \mathrm{CH}_{3}\right){ }_{2}^{13} \mathrm{CO} \ldots \mathrm{HF}$ рассчитаны по теории возмущений с использованием $a b$ initio методов МР2/6$311++\mathrm{G}(2 \mathrm{~d}, 2 \mathrm{p})$ и $\mathrm{MP} 2 / 6-311++\mathrm{G}(3 \mathrm{df}, 3 \mathrm{pd})$ с учетом ошибки наложения базисных функций мономеров. Результаты расчетов частот спектральных переходов находятся в хорошем согласии с экспериментальными данными и подтверждают обнаруженные в эксперименте эффекты изотопного замещения и образования водородной связи. Влияние ангармонических взаимодействий на частоты и интенсивности наиболее интенсивных полос $v(\mathrm{H}-\mathrm{F})$ и $v\left({ }^{13} \mathrm{C}=\mathrm{O}\right)$ изучено при помощи вариационных расчетов. Показано, что в отличие от комплекса $\left({ }^{12} \mathrm{CH}_{3}\right)_{2}^{12} \mathrm{CO} \ldots$. HF в комплексе $\left({ }^{12} \mathrm{CH}_{3}\right)_{2}^{13} \mathrm{CO} \ldots$. HF peзонанс $2 v(\mathrm{C}=\mathrm{O}) / v(\mathrm{H}-\mathrm{F})$ практически отсутствует.

Работа выполнена при финансовой поддержке Российского фонда фундаментальных исследований, грант № 18-03-00520. Исследования проведены с использованием оборудования ресурсного центра Научного парка СПбГУ „Геомодель“.

\section{Список литературы}

[1] Isotope Effects in Chemistry and Biology / Ed. by Kohen A., Limbach H.-H. Taylor \& Francis, 2006.

[2] Bulychev V.P., Buturlimova M.V., Tokhadze I.K., Tokhadze K.G. // J. Phys. Chem. A. 2014. V. 118. P. 7139.

[3] Bulychev V.P., Tokhadze K.G. // J. Mol. Struct. 2010. V. 976. P. 255.

[4] Arnold J., Millen D.J. // J. Chem. Soc. 1965. P. 510.

[5] Couzi M., Calve J., Huong P.V., Lascome J. // J. Mol. Struct. 1970. V. 5. P. 363.

[6] Andrews L., Johnson G.L. // J. Phys. Chem. 1984. V. 88. P. 5887.

[7] Bulychev V.P., Svishcheva E.A., Tokhadze K.G. // Spectrochim. Acta A. 2014. V. 117. P. 679.

[8] Bouteiller Y., Latajka Z. // J. Chem. Phys. 1992. V. 97. P. 45.

[9] Bouteiller Y., Latajka Z. // J.Mol. Struct. 1994. V. 322. P. 175.

[10] Gaussian 16, Revision A.03. Frisch M.J., Trucks G.W., Schlegel H.B., Scuseria G.E., Robb M.A., Cheeseman J.R., Scalmani G., Barone V., Petersson G.A., Nakatsuji H., Li X., Caricato M., Marenich V., Bloino J., Janesko B.G., Gomperts R., Mennucci B., Hratchian H.P., Ortiz J.V., Izmaylov A.F., Sonnenberg J.L., Williams-Young D., Ding F., Lipparini F., Egidi F., Goings J., Peng B., Petrone A., Henderson T., Ranasinghe D., Zakrzewski V.G., Gao J., Rega N., Zheng G., Liang W., Hada M., Ehara M., Toyota K., Fukuda R., Hasegawa J., Ishida M., Nakajima T., Honda Y., Kitao O., Nakai H., Vreven T., Throssell K., Montgomery J.A., Jr., Peralta J.E., Ogliaro F., Bearpark M.J., Heyd J.J., Brothers E.N., Kudin K.N., Staroverov V.N., Keith T.A., Kobayashi R., Normand J., Raghavachari K., Rendell A.P., Burant J.C., Iyengar S.S., Tomasi J., Cossi M., Millam J.M., Klene M., Adamo C., Cammi R., Ochterski J.W., Martin R.L., Morokuma K., Farkas O., Foresman J.B., Fox D.J. Gaussian, Inc., Wallingford CT, 2016. 
[11] Barone V. // J. Chem. Phys. 2005. V. 122. P. 014108.

[12] Bloino J., Barone V. // J. Chem. Phys. 2012. V. 136. P. 124108.

[13] Bloino J. // J. Phys. Chem. A. 2015. V. 119. P. 5269.

[14] Bulychev V.P., Buturlimova M.V., Tokhadze K.G. // J. Chem. Phys. 2018. V. 149. P. 104306.

[15] Булычев В.П., Мильке 3., Тохадзе К.Г., Уткина С.С. // Опт. и спектр. 1999. Т. 86. В. 3. С. 403.

[16] Bulychev V.P., Buturlimova M.V., Tokhadze K.G. // J. Phys. Chem. A. 2013. V. 117. P. 9093.

[17] Булычев В.П., Кочеварников А.М., Тохадзе К.Г. // Опт. и спектр. 2017. Т. 122. В. 6. С. 883.

[18] Bulychev V.P., Gromova E.I., Tokhadze K.G. // J. Phys. Chem. A. 2008. V. 112. P. 1251. 